\title{
Transtornos neurocognitivos e demência relacionados ao HIV em pessoas que fazem uso de antirretroviral: uma revisão integrativa
}

HIV-related neurocognitive disorders and dementia in people using antiretroviral: an integrative review

Trastornos neurocognitivos relacionados con el VIH y demencia en personas que utilizan antirretrovirales: una revisión integradora

\author{
Roni Robson da Silva \\ ORCID: https://orcid.org/0000-0001-6010-6438 \\ Universidade Veiga de Almeida, Brasil \\ E-mail: rr.roni1@gmail.com \\ Leandro Andrade da Silva \\ ORCID: https://orcid.org/0000-0003-3213-5527 \\ Universidade Veiga de Almeida, Brasil \\ E-mail: proflandrade@gmail.com \\ Maria Virginia Godoy da Silva \\ ORCID: https://orcid.org/0000-0003-3980-042X \\ Universidade Veiga de Almeida, Brasil \\ E-mail: godoydasilva@terra.com.br \\ Michael Silva Araujo \\ ORCID: https://orcid.org/0000-0002-5389-5712 \\ Universidade Cândido Mendes, Brasil \\ E-mail: michael_s.a@icloud.com \\ Milena Preissler das Neves \\ ORCID: https://orcid.org/0000-0002-3890-924X \\ Universidade Veiga de Almeida, Brasil \\ E-mail: milenapreissler@ gmail.com \\ Marcus Vinicius Lessa de Souza \\ ORCID: https://orcid.org/0000-0002-3023-4778 \\ Universidade Veiga de Almeida, Brasil \\ E-mail: contato.marcuslessa@gmail.com \\ Elson Santos de Oliveira \\ ORCID: https://orcid.org/0000-0001-9377-0140 \\ Universidade Veiga de Almeida, Brasil \\ E-mail: elson.oliveira@uva.br \\ Milton Domingues da Silva Junior \\ ORCID: https://orcid.org/0000-0003-1163-9005 \\ Universidade Veiga de Almeida, Brasil \\ E-mail: milton.enf.saude@gmail.com \\ Mônica Barreto Ribeiro \\ ORCID: https://orcid.org/0000-0001-8249-5622 \\ Centro Universitário Celso Lisboa, Brasil \\ E-mail: monicabarretobr@gmail.com
}

\begin{abstract}
Resumo
Distúrbios neurológicos e comprometimento cognitivo são comumente associados ao HIV. Embora o comprometimento neurocognitivo grave e progressivo tenha se tornado raro em clínicas de infectologia na era da terapia antirretroviral TARV potente, a maioria das PVHIV em todo o mundo apresenta desempenho abaixo das expectativas em testes neurocognitivos formais. Objetivo: Evidenciar os efeitos do HIV associado ao comprometimento neurocognitivo. Método: Trata-se de uma revisão integrativa da literatura que seguiu a estratégia PICO para identificar os fatores associados ao Transtorno Neurocognitivo relacionados ao HIV. A busca dos artigos foi realizada em três bases de dados eletrônicas: Medline, Lilacs e Scielo. Foram utilizados os descritores "Complexo AIDS Demência", "HIV", "Sobreviventes de Longo Prazo ao HIV", "Demência", "Transtornos Neurocognitivos", com textos completos, publicados no período de 2012 a 2022, no idioma inglês, português e espanhol e foi usado o operador booleano AND. Resultado: Foram encontrados 356 artigos. Vinte e cinco artigos foram selecionados para serem lidos na íntegra e 5 atenderam aos critérios desta revisão. Conclusão: As comorbidades contribuem para o comprometimento, mas são insuficientes para explicar a frequência do comprometimento encontrado. Os marcadores de doença do HIV, como a
\end{abstract}


carga viral atual e a contagem de CD4, não estão mais fortemente associados ao comprometimento contínuo da terapia, enquanto os marcadores de doença cardiovascular e os marcadores inflamatórios parecem mais associados. Novos biomarcadores de líquido cefalorraquidiano e neuroimagem são necessários para detectar e acompanhar o comprometimento. Pesquisa em andamento para otimizar a terapia do HIV no sistema nervoso central, e potencialmente intervir nos mecanismos de neurotoxicidade a jusante continuam sendo importantes avenidas de investigação futura. Em última análise, o controle total do vírus no cérebro é um passo necessário para a erradicação do HIV.

Palavras-chave: Complexo AIDS demência; HIV; Sobreviventes de longo prazo ao HIV; Demência; Transtornos neurocognitivos.

\begin{abstract}
Neurological disorders and cognitive impairment are commonly associated with HIV. Although severe and progressive neurocognitive impairment has become rare in infectious disease clinics in the era of potent antiretroviral therapy, the majority of HIV patients worldwide perform below expectations on formal neurocognitive tests. Objective: To demonstrate the effects of HIV associated with neurocognitive impairment. Method: This is an integrative literature review that followed the PICO strategy to identify factors associated with HIV-related Neurocognitive Disorder. The search for articles was carried out in three electronic databases: Medline, Lilacs and Scielo. The descriptors "AIDS Dementia Complex", "HIV", "Long-Term HIV Survivors", "Dementia", "Neurocognitive Disorders", with full texts, published from 2012 to 2022, in English, Portuguese and Portuguese, were used. Spanish and the Boolean operator AND was used. Result: 356 articles were found. Twenty-five articles were selected to be read in full and 5 met the criteria of this review. Conclusion: Comorbidities contribute to impairment, but are insufficient to explain the frequency of impairment found. HIV disease markers, such as current viral load and CD4 count, are not more strongly associated with continued commitment to therapy, while cardiovascular disease markers and inflammatory markers appear to be more associated. Novel cerebrospinal fluid and neuroimaging biomarkers are needed to detect and follow up on impairment. Ongoing research to optimize HIV therapy in the central nervous system, and potentially intervene in downstream neurotoxicity mechanisms remain important avenues for future investigation. Ultimately, total control of the virus in the brain is a necessary step towards the eradication of HIV.
\end{abstract}

Keywords: AIDS dementia complex; HIV; HIV long-term survivors; Dementia; Neurocognitive disorders.

\title{
Resumen
}

Los trastornos neurológicos y el deterioro cognitivo se asocian comúnmente con el VIH. Aunque el deterioro neurocognitivo severo y progresivo se ha vuelto raro en las clínicas de enfermedades infecciosas en la era de la terapia antirretroviral potente, la mayoría de los pacientes con VIH en todo el mundo se desempeñan por debajo de las expectativas en las pruebas neurocognitivas formales. Objetivo: Demostrar los efectos del VIH asociados al deterioro neurocognitivo. Método: Esta es una revisión integrativa de la literatura que siguió la estrategia PICO para identificar factores asociados con el Trastorno Neurocognitivo relacionado con el VIH. La búsqueda de artículos se realizó en tres bases de datos electrónicas: Medline, Lilacs y Scielo. Se utilizaron los descriptores "AIDS Dementia Complex", "HIV”, "Long-Term HIV Survivors", "Dementia", "Neurocognitive Disorders", con textos completos, publicados entre 2012 y 2022, en inglés, portugués y portugués. y se utilizó el operador booleano AND. Resultado: se encontraron 356 artículos. Veinticinco artículos fueron seleccionados para ser leídos en su totalidad y 5 cumplieron con los criterios de esta revisión. Conclusión: las comorbilidades contribuyen al deterioro, pero son insuficientes para explicar la frecuencia del deterioro encontrado. Los marcadores de la enfermedad del VIH, como la carga viral actual y el recuento de CD4, no están más fuertemente asociados con el compromiso continuo con la terapia, mientras que los marcadores de la enfermedad cardiovascular y los marcadores inflamatorios parecen estar más asociados. Se necesitan nuevos biomarcadores de neuroimagen y líquido cefalorraquídeo para detectar y hacer un seguimiento del deterioro. La investigación en curso para optimizar la terapia del VIH en el sistema nervioso central y potencialmente intervenir en los mecanismos de neurotoxicidad aguas abajo siguen siendo vías importantes para la investigación futura. En última instancia, el control total del virus en el cerebro es un paso necesario hacia la erradicación del VIH.

Palabras clave: Complejo SIDA demencia; VIH; Sobrevivientes de VIH a largo plazo; Demencia; Trastornos neurocognitivos.

\section{Introdução}

O vírus da imunodeficiência humana (HIV) surgiu como um grande desafio para a saúde mundial há quase 40 anos e desafiou cientistas e clínicos a combater seu vasto e devastador impacto (Pulliam et al., 2019). Embora reconhecido por seu impacto direto no sistema imunológico através da depleção de linfócitos CD4, também teve um amplo impacto no sistema nervoso, incluindo evidências de patologia direta no cérebro, medula espinhal e nervos periféricos (Banerjee et al., 2019; Ru \& Tang, 2017). O transtorno neurocognitivo associado ao HIV (TNAH) combinado com um espectro único de infecções oportunistas, compreende a neuro AIDS (Han et al., 2018). 
O cenário da neuro AIDS evoluiu rapidamente impulsionado pelas opções terapêuticas emergentes disponíveis para o atendimento de pacientes com HIV (Abassi et al., 2017). O desenvolvimento da terapia antirretroviral combinada (TARV) transformou o HIV em uma doença crônica, com expectativa de vida próxima das normas da população para os pacientes aderentes aos medicamentos (Liu et al., 2014). As principais questões remanescentes incluem as implicações do baixo nível persistente de HIV, respostas inflamatórias contínuas, potencial toxicidade terapêutica e interação entre envelhecimento e neuro degeneração devido ao HIV (Han et al., 2018). Uma cura funcional das pessoas vivendo com HIV (PVHIV) exigirá o silenciamento do vírus em todos os compartimentos do corpo, incluindo o cérebro (Rosenthal \& Tyor, 2019).

O HIV continua a ser mais prevalente nos países em desenvolvimento (Sacktor, 2018). Como a TARV geralmente é iniciada tardiamente nesses locais, as infecções oportunistas neurológicas, particularmente meningite criptocócica e tuberculosa, encefalite por toxoplasma e leucoencefalopatia multifocal progressiva se manifestam (Abassi et al., 2017). Essas complicações são amplamente silenciadas após a obtenção da TARV estável (Silva et al., 2019). Além disso, a neuropatia periférica pode impactar na qualidade de vida dos pacientes com HIV, mas é reduzida quando a terapia que evita os antirretrovirais neurotóxicos é iniciada logo após a infecção (Rubin et al., 2019).

Muito aquém da busca pela cura, o progresso para eliminar a deficiência neurológica associada ao HIV tem sido desencorajador (Troncoso \& Conterno, 2015). Estudos transversais continuam demonstrando que cerca de metade de todos as PVHIV tratados apresentam comprometimento cognitivo (De Souza Francisco Barbosa et al., 2021). Um aspecto positivo da era TARV é que as formas mais graves de comprometimento neurocognitivo diminuíram, embora as formas mais leves permaneçam (Neves et al., 2019). Classificar a etiologia, o prognóstico e o regime ideal de TARV para pacientes tratados com TNAH continua sendo uma grande tarefa incompleta (Mackiewicz et al., 2019). Compreender as definições atuais de TNAH é essencial. A introdução da TARV resultou em benefícios claros. Como resultado da reconstituição imune, as condições oportunistas tornaram-se raras e a TNAH agora raramente se desenvolve (Ances \& Letendre, 2019). No entanto, existe variabilidade na penetração e transporte de medicamentos antirretrovirais através da barreira hematoencefálica (Namagga et al., 2019). Isso levou a sérias preocupações sobre o cérebro servir como um foco de infecção que poderia arder com controle parcial. Em teoria, o vírus resistente poderia evoluir no sistema nervoso central e semear novamente no corpo (Eggers et al., 2017). A eliminação do vírus do sistema nervoso central (SNC) será necessária para alcançar a cura (Rahimian \& He, 2017).

Uma definição de pesquisa de consenso foi articulada em 2006 em Frascati, Itália com subclassificações TNAH criadas que incluem comprometimento neurocognitivo assintomático (CNA), transtorno neurocognitivo leve (TNL) e demência associada ao HIV (DAH) (Gelman et al., 2013). Independente de condições oportunistas, o HIV avançado está associado ao comprometimento cognitivo, consequência da infecção pelo HIV no sistema nervoso (Silva et al., 2020). "AIDS Dementia Complex" (ADC), uma demência subcortical. foi caracterizada como uma condição incapacitante progressiva que se manifestava por perda crescente de atenção e concentração, lentidão motora acentuada e componentes comportamentais variáveis, geralmente levando à morte em menos de um ano (Sacktor et al., 2016). Essa síndrome foi associada a alterações patológicas no cérebro que incluem atrofia generalizada, alterações na substância branca causando leucoencefalopatia, nódulos microgliais típicos de encefalite viral e células gigantes multinucleadas que parecem estar diretamente infectadas pelo HIV, identificadas por coloração de antígeno (Mohamed et al., 2020). Na infecção não tratada, a gravidade da demência foi mais intimamente associada aos marcadores de resposta inflamatória do que à carga viral, embora a carga viral do líquido cefalorraquidiano (LCR) tenha sido modestamente associada às manifestações clínicas (Illenberger et al., 2020). O comprometimento progressivo descrito como ADC, agora é referido como Demência Associada ao HIV (DAH) usando critérios recentes (Vázquez-Santiago et al., 2014).

No entanto, a confusão ainda pode permanecer, uma vez que o termo DAH é aplicado não apenas à doença cerebral progressiva devido à AIDS não tratada, mas também a pessoas que têm comprometimento neurológico residual associado ao HIV substancial (Elbirt et al., 2015). Não é inesperado que a população significativa e crescente que teve lesão cerebral devido 
ao HIV, mas não tem uma doença progressiva óbvia, não seja ajudada pelos mesmos tratamentos que aqueles com patologia mediada por vírus ativa (Da Silva et al., 2021). Extensas pesquisas em neuro AIDS não conseguiram separar essas populações, contribuindo para avaliações decepcionantes ou confusas (Clifford \& Ances, 2013). A aplicação dos atuais critérios TNAH baseou-se muito em fazer um diagnóstico de acordo com a gravidade do comprometimento neurológico no exame neuropsicológico (Gavegnano et al., 2019). O Objetivo desse estudo foi buscar na literatura cientifica mundial o que vem sendo produzido bem como o estado da arte sobre essa temática dos transtornos neuro cognitivos causados pelo HIV.

\section{Metodologia}

Trata-se de uma revisão integrativa da literatura. Método que se caracteriza por reunir e sintetizar resultados de pesquisas sobre um tema, de maneira sistemática e ordenada (De Souza Francisco Barbosa et al., 2021). A pergunta de pesquisa foi definida a partir da estratégia PICO, que prevê a definição do participante (P), intervenção (I), comparação (C) e desfecho/ outcomes (O). Pretende-se responder à questão norteadora: Quais fatores identificados na literatura $(\mathrm{O})$, estão associados ao transtorno neucognitivo (I) em pessoas vivendo com HIV (P)? Em seguida, as palavras-chave "Complexo AIDS Demência", "HIV", "Sobreviventes de Longo Prazo ao HIV", "Demência", "Transtornos Neurocognitivos", foram definidas a partir do vocabulário dos Descritores em Ciências da Saúde (DeCS), por ser uma terminologia comum à pesquisa. Essas, foram combinadas entre si, utilizando-se o operador booleano AND nas bases de dados e/ou bibliotecas eletrônicas: Medical Literature Analysis and Retrieval System Online (MEDLINE), da Literatura Latino-Americana e do Caribe em Ciências da Saúde (LILACS) e da Scientific Eletronic Library Online (SciELO). A mesma estratégia de busca foi realizada em todas as bases de dados e/ ou bibliotecas eletrônicas. Os critérios de inclusão dos artigos para análise foram: grupo populacional de pessoas que vivem com HIV e fazem uso de TARV, publicados entre 2012 e 2022, disponíveis na íntegra, nos idiomas português, inglês e espanhol, que tratavam sobre a temática de estresse de minoria de gênero sexual. Foram excluídos artigos de opinião, editoriais, outras revisões, artigos duplicados e publicações que não tratassem da temática. O período de coleta ocorreu nos meses de novembro 2021 a janeiro de 2022. Para a análise dos dados, foi construído um quadro analítico (Quadro 2) que possibilitou reunir e sintetizar as informações-chave dos estudos. O instrumento de coleta reuniu as seguintes informações: título, autor(es)/ano de publicação/país, objetivo, método, principais resultados. O nível de evidência identificado nos artigos analisados foi classificado de acordo com o sistema Grading of Recommendations Assessment, Development and Evaluation (GRADE) (Gendelman \& Gelbard, 2014), um sistema considerado sensível para graduar a qualidade das evidências. Nesse sistema, a qualidade da evidência é descrita em quatro níveis: alto, moderado, baixo e muito baixo (Quadro 1). A evidência proveniente de ensaios clínicos randomizados começa com nível alto e a evidência proveniente de estudos observacionais, com nível baixo (Gendelman \& Gelbard, 2014).

Quadro 1 - Níveis de evidência, Rio de Janeiro 2022.

\begin{tabular}{|c|l|l|}
\hline Nível & \multicolumn{1}{|c|}{ Definição } & \multicolumn{1}{c|}{ Implicações } \\
\hline Alto & $\begin{array}{l}\text { Há forte confiança de que o verdadeiro } \\
\text { efeito esteja próximo daquele estimado }\end{array}$ & $\begin{array}{l}\text { É improvável que trabalhos adicionais irão modificar a } \\
\text { confiança na estimativa do efeito }\end{array}$ \\
\hline Moderado & $\begin{array}{l}\text { Há confiança moderada no efeito } \\
\text { estimado }\end{array}$ & $\begin{array}{l}\text { Trabalhos futuros poderão modificar a confiança na } \\
\text { estimativa de efeito, com possibilidade, inclusive, de } \\
\text { modificar a estimativa }\end{array}$ \\
\hline Baixo & A confiança no efeito é limitada & $\begin{array}{l}\text { Trabalhos futuros provavelmente terão um impacto } \\
\text { importante em nossa confiança na estimativa de efeito }\end{array}$ \\
\hline Muito Baixo & $\begin{array}{l}\text { A confiança na estimativa de efeito é } \\
\text { muito limitada. Há importante grau de } \\
\text { incerteza nos achados }\end{array}$ & Qualquer estimativa de efeito é incerta \\
\hline
\end{tabular}




\section{Resultados e Discussão}

Identificou-se, um total de 356 estudos nas referidas bases de dados, conforme ilustrado na (Figura 1), a qual seguiu as recomendações PRISMA (Page et al., 2021) para descrever o processo de busca na literatura. Desses, foram excluídos 131 artigos duplicados, restando 225 artigos únicos. Em seguida, realizou-se a leitura dos títulos e resumos observando-se os critérios de inclusão e exclusão. Como resultado desse processo, 200 artigos foram excluídos e outros 25 artigos se adequaram aos critérios de elegibilidade. Iniciou-se, então, a leitura integral e em profundidade desses estudos por dois revisores, independentemente. Eventuais discordâncias entre os avaliadores, surgidas durante esse estágio, foram trabalhadas e resolvidas mediante consenso, o que resultou em uma amostra final de 5 artigos. Os artigos incluídos nesta síntese (Figura 1) foram desenvolvidos em seis diferentes países: Estados Unidos $(n=1)$, Inglaterra $(n=1)$, Quênia $(n=1)$, Canada $(n=1)$ Alemanha $(n=1)$.

Quanto ao método, a maioria dos pesquisadores utilizou a abordagem quantitativa $(n=3)$ para descrever e analisar, em profundidade, as diferentes dimensões em que ocorre a inserção de pessoas transgênero na sociedade. Outros 2 trabalhos foram estudos de revisão, e em apenas um dos estudos, os autores indicaram fazer uso de métodos quantitativos e qualitativos, complementarmente. Embora esse tipo de desenho metodológico tenha se mostrado o mais apropriado para desvelar as variadas facetas da sexualidade e das relações humanas e sociais, este fato caracteriza a totalidade dos artigos como sendo de baixo de nível de evidência (Galvão \& Pereira, 2015). A prevalência de TNAH atualmente é impulsionada por CNA e TNL. As categorias são baseadas em anormalidades de testes neuropsicométricos com ou sem prejuízo funcional nas atividades da vida diária. É claro que, em comparação com as melhores normas populacionais disponíveis, cerca de metade das PVHIV continuam a ter níveis de desempenho mais baixos do que seria previsto (W. R. Tyor \& Bimonte-Nelson, 2018). No entanto, as definições atuais da pesquisa TNAH para CNA e TNL podem ser excessivamente inclusivas, resultando em uma inflação clinicamente sem importância dos números de deficiência (Silva, Pontes, et al., 2021). Com base no desempenho neuropsicométrico que carece de normas idealmente compatíveis, conhecimento do desempenho anterior e exclusão de uma infinidade de questões de confusão, a utilidade da categoria CNA permanece controversa (Geffin \& McCarthy, 2018). 
Figura 1 - Seleção dos artigos por descritores nas bases de dados, Rio de Janeiro 2022.
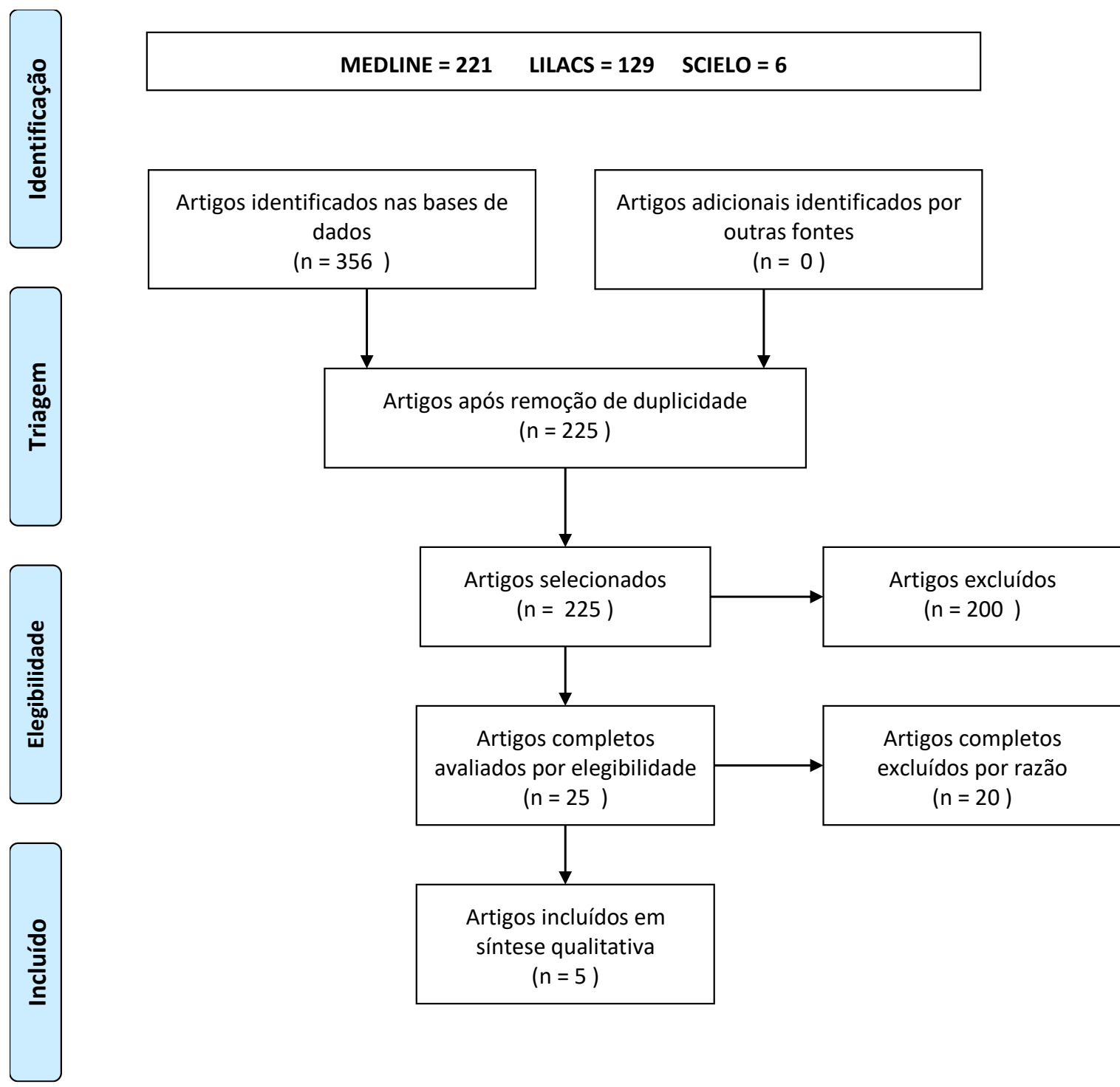

Fonte: Autores (2022).

Confundindo ainda mais os critérios atuais é a definição de comprometimento funcional, a característica que distingue CNA de TNL (Silva, Silva, et al., 2021). Na prática, a avaliação do comprometimento funcional mostra-se desafiadora e provavelmente imprecisa (Geffin \& McCarthy, 2018). Uma variedade de abordagens é permitida para definir a capacidade funcional (Debalkie Animut et al., 2019). Normalmente, o autorrelato tem sido usado, mas isso é subjetivo. Se o comprometimento funcional for observado, muitas vezes é difícil distinguir se as alterações são causadas pela infecção pelo HIV ou outras etiologias (Souza et al., 2021). 
Quadro 2 - Categorização da produção científica incluída na revisão, Rio de Janeiro 2022.

\begin{tabular}{|c|c|c|c|c|c|}
\hline Títulos & $\begin{array}{c}\text { Autor(es), } \\
\text { País/ Ano }\end{array}$ & Objetivo & Método & Resultados & $\begin{array}{c}\text { Nível de } \\
\text { Evidência }\end{array}$ \\
\hline $\begin{array}{l}\text { HIV-1-associated } \\
\text { neurocognitive } \\
\text { disorder: } \\
\text { epidemiology, } \\
\text { pathogenesis, } \\
\text { diagnosis, and } \\
\text { treatment }\end{array}$ & $\begin{array}{c}\text { Eggers C, } \\
\text { Arendt G, } \\
\text { Hahn K, et al, } \\
2017 \text { / EUA }\end{array}$ & $\begin{array}{l}\text { Trazer panorama da } \\
\text { visão geral dos } \\
\text { conceitos atuais no } \\
\text { campo do transtornos } \\
\text { neurocognitivo } \\
\text { associado ao hiv e } \\
\text { fornece sugestões para o } \\
\text { manejo diagnóstico e } \\
\text { terapêutico }\end{array}$ & $\begin{array}{c}\text { Revisão } \\
\text { Sistematica }\end{array}$ & $\begin{array}{l}\text { A idade crescente da } \\
\text { população infectada pelo HIV } \\
\text { tornará cada vez mais } \\
\text { necessária a inclusão de } \\
\text { formas de demência não } \\
\text { associadas ao HIV nos } \\
\text { diagnósticos diferenciais de } \\
\text { TNAH. }\end{array}$ & Alto \\
\hline $\begin{array}{c}\text { HIV-1 } \\
\text { Encephalopathy } \\
\text { And Aids } \\
\text { Dementia } \\
\text { Complex }\end{array}$ & $\begin{array}{c}\text { Kopstein, } \\
\text { Mohlman, } \\
2020 \text { / } \\
\text { Inglaterra }\end{array}$ & $\begin{array}{l}\text { Identificar a etiologia } \\
\text { dos distúrbios } \\
\text { neurocognitivos } \\
\text { associados ao HIV } \\
\text { (TNAH). }\end{array}$ & $\begin{array}{c}\text { Estudo } \\
\text { transversal }\end{array}$ & $\begin{array}{l}\text { Uma avaliação neurológica } \\
\text { pode ser necessária para } \\
\text { completar totalmente a } \\
\text { investigação para causas } \\
\text { alternativas de déficits } \\
\text { cognitivos. }\end{array}$ & Baixo \\
\hline $\begin{array}{l}\text { HIV-associated } \\
\text { neurocognitive } \\
\text { disorders at Moi } \\
\text { teaching and } \\
\text { referral hospital, } \\
\text { Eldoret, Kenya }\end{array}$ & $\begin{array}{c}\text { Mohamed, } \\
\text { Oduor, } \\
\text { Kinyanjui, } \\
2020 \text { / Kenya }\end{array}$ & 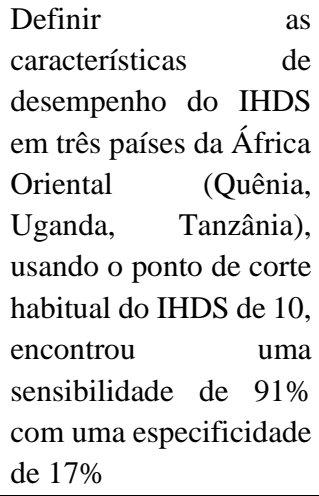 & $\begin{array}{c}\text { Estudo } \\
\text { transversal }\end{array}$ & $\begin{array}{l}\text { A prevalência de Transtorno } \\
\text { Neurocognitivos Associados } \\
\text { ao HIV permanece alta neste } \\
\text { erro de Terapia Antirretroviral } \\
\text { Altamente Ativa com maior } \\
\text { prevalência de TNAH leve } \\
(78,6 \%) \text { e baixa prevalência de } \\
\text { TNAH grave }(2,5 \%) \text {. }\end{array}$ & Moderado \\
\hline $\begin{array}{l}\text { HIV Infection } \\
\text { and } \\
\text { Neurocognitive } \\
\text { Disorders in the } \\
\text { Context of } \\
\text { Chronic Drug } \\
\text { Abuse: Evidence } \\
\text { for Divergent } \\
\text { Findings } \\
\text { Dependent upon } \\
\text { Prior Drug } \\
\text { History }\end{array}$ & $\begin{array}{c}\text { Illenberger et } \\
\text { al., } 2020 \\
\text { / Canada }\end{array}$ & $\begin{array}{l}\text { A presente revisão irá, } \\
\text { portanto, explorar como } \\
\text { o circuito fronto- } \\
\text { estriatal se adapta ao } \\
\text { uso de drogas (usando } \\
\text { cocaínar como } \\
\text { exemplo), infecção por } \\
\text { HIV-1 }\end{array}$ & $\begin{array}{c}\text { Revisão } \\
\text { Sistemática }\end{array}$ & $\begin{array}{l}\text { Nós postulamos que os efeitos } \\
\text { do uso de drogas e da infecção } \\
\text { pelo HIV-1 na microglia } \\
\text { interagem para impulsionar a } \\
\text { progressão da disfunção } \\
\text { motivacional em um ritmo } \\
\text { acelerado. }\end{array}$ & Baixo \\
\hline $\begin{array}{c}\text { Impact of } \\
\text { minocycline on } \\
\text { cerebrospinal } \\
\text { fluid markers of } \\
\text { oxidative stress, } \\
\text { neuronal injury, } \\
\text { and inflammation } \\
\text { in HIV- } \\
\text { seropositive } \\
\text { individuals with } \\
\text { cognitive } \\
\text { impairment }\end{array}$ & $\begin{array}{c}\text { Sacktor et al., } \\
2014 \text { / } \\
\text { Alemanha }\end{array}$ & $\begin{array}{l}\text { O objetivo deste estudo } \\
\text { foi examinar o efeito da } \\
\text { minociclina em } \\
\text { marcadores de estresse } \\
\text { oxidativo, lesão } \\
\text { neuronal, níveis de } \\
\text { neurotransmissores, e } \\
\text { inflamação do LCR em } \\
\text { participantes em } \\
\text { A5235. }\end{array}$ & $\begin{array}{l}\text { Estudo de } \\
\text { coorte }\end{array}$ & $\begin{array}{l}\text { Em última análise, será } \\
\text { necessária a identificação de } \\
\text { um conjunto de biomarcadores } \\
\text { específicos para obter um } \\
\text { diagnóstico precoce e preciso, } \\
\text { o que será necessário } \\
\text { assumindo que tratamentos } \\
\text { específicos para TNAH sejam } \\
\text { desenvolvidos. }\end{array}$ & $\begin{array}{l}\text { Muito } \\
\text { Baixo }\end{array}$ \\
\hline
\end{tabular}


O Multicenter AIDS Cohort Study (MACS) estudou o desempenho neurocognitivo de pacientes HIV assintomáticos que estavam imunologicamente intactos ou virologicamente controlados e indivíduos soronegativos para HIV durante um intervalo de cinco anos (Illenberger et al., 2020). Os pacientes com HIV não apresentaram declínio em vários testes neuropsicométricos (Silva \& Silva, 2021). Esta observação é importante e tranquilizadora (W. Tyor et al., 2013). Os testes foram sensíveis o suficiente para detectar o declínio antecipado relacionado à idade, mas não conseguiram demonstrar que houve maior deterioração no desempenho para a população soropositiva assintomática, mesmo em indivíduos com controle viral imperfeito (Thaney \& Kaul, 2019). Por outro lado, existem inúmeros relatos de que alguns pacientes com HIV continuam a apresentar deterioração neurocognitiva, apesar da terapia virologicamente bem-sucedida (Sacktor, 2018). A capacidade de avaliar as funções neurológicas exige uma avaliação cuidadosa e, em ambientes clínicos movimentados, condições leves podem ser negligenciadas (Rosenthal \& Tyor, 2019). As observações longitudinais do CNS HIV Antiretroviral Therapy Effects Research (CHARTER) de uma grande coorte clínica acadêmica dos EUA foram relatadas de forma preliminar (Abassi et al., 2017). Desempenho neurocognitivo, medidas laboratoriais e exames neurológicos foram realizados a cada 6 meses em um intervalo de 42 meses (Ances \& Letendre, 2019). A maioria (61\%) dos pacientes com HIV permaneceu estável, enquanto 16,5\% aparentemente melhoraram o estado neurocognitivo e 22,7\% diminuíram (Neves et al., 2019). Os fatores associados ao risco de progressão incluíram a presença de comorbidades graves (como outras infecções, abuso de drogas, outras condições neurológicas) ou evidência de falha na terapia do HIV (sem TARV e/ou baixa contagem de CD4) (Gelman et al., 2013).

Ensaios clínicos envolvendo pacientes HIV com TNAH que recebem controle ou intervenção reforçaram a impressão de que as alterações cognitivas devido ao HIV, se presentes, devem ser lentas, uma vez que normalmente não ocorrem em braços de controle em ensaios de curta duração (Banerjee et al., 2019). No entanto, na era TARV, o uso de testes de desempenho neuropsicométricos é mais desafiador, pois a principal preocupação é identificar pacientes com déficits mais sutis (Han et al., 2018). A deteç̧ão de déficits leves requer testes mais difíceis que geralmente levam mais tempo em comparação com o motor temporizado simples administrado (Gavegnano et al., 2019).

Os testes neuropsicométricos devem continuar a ser utilizados para avaliar a eficácia das intervenções para o desempenho durante os ensaios terapêuticos (Gendelman \& Gelbard, 2014). Quando os pacientes servem como seus próprios controles e são comparados a outros pacientes monitorados de forma semelhante pela mesma equipe de investigação ao longo do tempo, essas medidas podem ser sensíveis para a detecção de alterações relevantes para intervenções terapêuticas (Geffin \& McCarthy, 2018). Grupos comparativos estudados com a mesma sequência de testes podem explicar os efeitos de aprendizado que podem ser difíceis de detectar sem controles simultâneos (Vázquez-Santiago et al., 2014).

A avaliação do LCR refletem a biologia do sistema nervoso central mais de perto do que o sangue (Troncoso \& Conterno, 2015). A barreira hematoencefálica limita o movimento do sangue para os compartimentos cerebrais, resultando em um compartimento fisiológico relativo (Namagga et al., 2019). Muitas vezes, pacientes e médicos relutam indevidamente em realizar uma punção lombar para coleta de LCR (Rahimian \& He, 2017). Na ausência de coagulação anormal ou grande massa assimétrica no cérebro, as punções lombares são extremamente seguras e, quando realizadas por médicos qualificados, geralmente não são tão nocivas quanto muitos outros procedimentos (Debalkie Animut et al., 2019). O uso de agulhas não cortantes reduz muito a chance de dores de cabeça ortostáticas transitórias que às vezes se seguem à punção lombar. Quando informações únicas podem ser coletadas, uma punção lombar deve ser realizada (Vázquez-Santiago et al., 2014).

É claro que ocasionalmente o HIV emerge no LCR anunciado por novos sintomas ou sinais neurológicos, mesmo diante do controle virológico sanguíneo contínuo (Geffin \& McCarthy, 2018). Esse fenômeno de "escape" viral é incomum, mas deve alertar o clínico para avaliar o LCR se houver sintomas neurológicos novos ou ativos, não explicados de outra forma (Da Silva et al., 2021). Raramente, pacientes assintomáticos também têm HIV detectável em baixo número de cópias quando os níveis virais no sangue estão bem controlados (Clifford \& Ances, 2013). Atualmente, há pouca evidência de evolução viral do LCR em 
pacientes HIV tratados com sucesso, mas são necessários estudos longitudinais adicionais do LCR em pacientes tratados (Han et al., 2018).

As características do vírus recuperados no LCR ou no SNC podem refletir características únicas de um vírus neurotrófico (Banerjee et al., 2019). A utilidade clínica dos marcadores inflamatórios no LCR tem sido limitada, mas essas medidas podem potencialmente identificar pacientes em risco de desenvolver TNAH (Liu et al., 2014). Mesmo os pacientes em uso de TARV supressora de longo prazo têm neopterina e índice de IgG levemente elevados no LCR. Marcadores de ativação imune persistentes, incluindo IL-6, IL-8, CCL2 (MCP-1) permanecem presentes em pacientes com TARV tratados com sucesso (Banerjee et al., 2019). Outra situação em que a resposta imune desregulada pode levar ao comprometimento e aos sintomas é durante a reconstituição imune, particularmente nos raros, mas dramáticos casos de encefalite CD8 que são relatados durante a infecção pelo HIV tratada (Ru \& Tang, 2017).

A evidência prática da supressão bem-sucedida do HIV a longo prazo na era TARV com apenas raro escape do SNC é tranquilizadora de que praticamente todas as combinações de drogas servem para controlar o vírus no compartimento do SNC (Pulliam et al., 2019). Contudo, esse risco teórico, juntamente com o reconhecimento de TNAH em andamento, inspirou apropriadamente a consideração intensiva e o monitoramento da terapia (Gavegnano et al., 2019). De fato, uma explicação para a prevalência contínua de TNAH é que a viremia de baixo nível no SNC pode continuar, conduzindo a neuro degeneração por ativação inflamatória tóxica e/ou produtos virais tóxicos, como a proteína tat.

Uma estratégia terapêutica alternativa enfatizou que os monócitos e macrófagos parecem ser o reservatório celular primário que afeta o SNC, representando células residentes com infecção proliferativa no cérebro não tratado e potencialmente abrigando o vírus em monócitos circulantes mesmo durante a terapia eficaz (Sacktor et al., 2016). Quando a terapia foi graduada por eficácia para infecção de monócitos, os resultados cognitivos pareciam correlacionados com esse índice. Uma investigação mais aprofundada desta estratégia merece consideração (Mohamed et al., 2020).

A demonstração de avanço viral em andamento em algumas amostras de LCR, bem como o reconhecimento de que níveis muito baixos de vírus são detectados no LCR se forem usados ensaios ultrassensíveis, também estimulou a consideração de regimes de intensificação do tratamento que incluem novas classes de medicamentos (W. R. Tyor \& Bimonte-Nelson, 2018). Prevê-se que os antagonistas de CCR5 contribuam exclusivamente para os isolados do SNC, enquanto os inibidores da integrase merecem uma avaliação adicional quanto à potência no SNC (Eggers et al., 2017). Embora pequenos ensaios de intensificação tenham sido decepcionantes até o momento, ensaios multicêntricos maiores poderiam abordar melhor essa possibilidade (Troncoso \& Conterno, 2015). A aplicação de nanopartículas para aumentar a entrega de antirretrovirais ao cérebro também está sob investigação, com a ressalva de que uma entrega mais eficaz também pode aumentar a toxicidade intrínseca (Namagga et al., 2019).

Um estudo recente testou a minociclina, que se acredita inibir a ativação microglial e ter propriedades antioxidantes e neuroprotetoras (Rahimian \& He, 2017). Em um modelo de vírus da imunodeficiência símia de encefalite, a minociclina reduziu a doença inflamatória cerebral (De Souza Francisco Barbosa et al., 2021). Nenhum benefício no estado neurocognitivo ou nos marcadores de doença pode ser observado em populações tratadas com antirretrovirais ou não tratadas com TNAH (Mackiewicz et al., 2019). Um estudo atual está investigando o fluconazol e um inibidor de recaptação de serotonina (ISRS) com base em modelos animais de TNAH e dados de coorte associados a ISRS (Gelman et al., 2013). O uso de medicamentos aprovados para a doença de Alzheimer foi considerado (Ances \& Letendre, 2019). A memantina, um antagonista de NMDA com propriedades neuroprotetoras in vitro, não demonstrou neuroproteção ou melhora cognitiva, enquanto um pequeno teste recente de um inibidor da acetilcolinesterase pareceu proporcionar uma leve melhora sintomática (Doyle et al., 2015). O ácido valpróico tem sido um fármaco de interesse para o HIV, mais recentemente como um inibidor da histona desacetilase que pode ser utilizado como ferramenta de ativação celular em uma estratégia de cura (Rubin et al., 2019). Também foi relatado que tem propriedades 
neuroprotetoras (Abassi et al., 2017). Um pequeno estudo mostrou uma tendência de melhora cognitiva usando testes neuropsicométricos e medidas de neuroimagem (Silva et al., 2019). No entanto, isso não foi comprovado em outro ensaio (Sacktor, 2018). Atualmente, as estratégias anti-inflamatórias são a terapia adjuvante mais discutida para TNAH (Rosenthal \& Tyor, 2019). As próximas avaliações de metotrexato em baixas doses, bem como grandes ensaios avaliando estatinas, podem fornecer oportunidades para investigar ainda mais o impacto da inflamação na função neurocognitiva (Sacktor, 2018).

Pesquisas sobre interações especiais entre mudanças antecipadas do envelhecimento e infecção crônica pelo HIV chamaram a atenção, principalmente em torno de possíveis interações de imunossenescência (Abassi et al., 2017). Foi considerada a possibilidade de que o comprometimento neurocognitivo do HIV resulte da expressão precoce de distúrbios cerebrais degenerativos, como a doença de Alzheimer (DA) causada pelo HIV (Ances \& Letendre, 2019). Enquanto a DAH na era antes TARV era característica da demência subcortical ao contrário da doença de Alzheimer, a apresentação clínica atual da TNAH pode ser semelhante à doença de Alzheimer (Troncoso \& Conterno, 2015). Alguns relatórios suportam achados patológicos consistentes com a doença de Alzheimer, mas uma confirmação patológica totalmente consistente não foi descrita (Mackiewicz et al., 2019). Alguns relatórios sugerem pelo menos uma sobreposição parcial nos biomarcadores do LCR para a doença de Alzheimer e TNAH (Rosenthal \& Tyor, 2019).

\section{Conclusão}

Parece importante que o estado dinâmico do comprometimento, bem como a fisiopatologia associada, seja incluído nos esforços diagnósticos e terapêuticos. Isso pode ajudar o campo a resolver os problemas restantes de forma mais eficaz. O envolvimento neurológico durante a infecção pelo HIV continua sendo um aspecto importante da infecção que requer estudo contínuo. Tanto os testes objetivos da função neurológica quanto o testemunho dos pacientes confirmam que a TARV, embora tenha melhorado imensamente os resultados, não alcançou a proteção funcional completa do sistema nervoso. Como as mudanças agora são sutis e, em geral, mudam lentamente, o TNAH continua sendo um desafio para o estudo, mas a importância da função cerebral para a independência e a qualidade de vida exige que esforços contínuos sejam direcionados para otimizar esse aspecto do atendimento a PVHIV. É quase certo que múltiplos mecanismos contribuem e, portanto, múltiplas intervenções terapêuticas precisarão ser racionalmente empregadas para alcançar o sucesso. Mais pesquisas em larga escala são necessárias para uma melhor compreensão desse evento. Uma limitação desse trabalho foi a baixa quantidade de estudos publicados.

\section{Referências}

Abassi, M., Morawski, B. M., Nakigozi, G., Nakasujja, N., Kong, X., Meya, D. B., Robertson, K., Gray, R., Wawer, M. J., Sacktor, N., \& Boulware, D. R. (2017). Cerebrospinal fluid biomarkers and HIV-associated neurocognitive disorders in HIV-infected individuals in Rakai, Uganda. Journal of Neurovirology, 23(3), 369-375. https://doi.org/10.1007/s13365-016-0505-9

Ances, B. M., \& Letendre, S. L. (2019). CROI 2019: neurologic complications of HIV disease. Topics in Antiviral Medicine, 27(1), $26-33$.

Banerjee, N., McIntosh, R. C., \& Ironson, G. (2019). Impaired Neurocognitive Performance and Mortality in HIV: Assessing the Prognostic Value of the HIVDementia Scale. AIDS and Behavior, 23(12), 3482-3492. https://doi.org/10.1007/s10461-019-02423-w

Clifford, D. B., \& Ances, B. M. (2013). HIV-associated neurocognitive disorder. The Lancet. Infectious Diseases, 13(11), 976-986. https://doi.org/10.1016/S1473-3099(13)70269-X

Da Silva, R. R., Lessa de Souza, M. V., Ferracini Alencar, I., Ferreira Leite Inácio, A., Ferreira da Silva, D., Ferro Messias, I., \& Lemos de Magalhães, A. F. (2021). Neuropatias diabéticas periféricas como complicações do diabetes mellitus: estudo de revisão. Saúde Coletiva (Barueri), 11(67), 6923-6936. https://doi.org/10.36489/saudecoletiva.2021v11i67p6923-6936

De Souza Francisco Barbosa, I., De Fátima de Sousa Mendonça, C., Andrade da Silva, L., Preissler das Neves, M., Da Silva, R. R., Ferracini de Alencar, I., \& Da Silva de Araújo, M. (2021). Produções acadêmicas sobre a assistência destinada às pessoas com problemas relacionados ao abuso de álcool. Um estudo de revisão. Saúde Coletiva (Barueri), 11(63), 5424-5435. https://doi.org/10.36489/saudecoletiva.2021v11i63p5424-5435

Debalkie Animut, M., Sorrie, M. B., Birhanu, Y. W., \& Teshale, M. Y. (2019). High prevalence of neurocognitive disorders observed among adult people living with HIV/AIDS in Southern Ethiopia: A cross-sectional study. PloS One, 14(3), e0204636. https://doi.org/10.1371/journal.pone.0204636 
Doyle, K. L., Morgan, E. E., Weber, E., \& Woods, S. P. (2015). Time estimation and production in HIV-associated neurocognitive disorders (HAND). Journal of the International Neuropsychological Society: JINS, 21(2), 175-181. https://doi.org/10.1017/S1355617715000089

Eggers, C., Arendt, G., Hahn, K., Husstedt, I. W., Maschke, M., Neuen-Jacob, E., Obermann, M., Rosenkranz, T., Schielke, E., \& Straube, E. (2017). HIV-1associated neurocognitive disorder: epidemiology, pathogenesis, diagnosis, and treatment. Journal of Neurology, 264(8), 1715-1727. https://doi.org/10.1007/s00415-017-8503-2

Elbirt, D., Mahlab-Guri, K., Bezalel-Rosenberg, S., Gill, H., Attali, M., \& Asher, I. (2015). HIV-associated neurocognitive disorders (HAND). The Israel Medical Association Journal : IMAJ, 17(1), 54-59.

Galvão, T. F., \& Pereira, M. G. (2015). Avaliação da qualidade da evidência de revisões sistemáticas. Epidemiologia e Serviços de Saúde, 24(1), 775-778. https://doi.org/10.5123/S1679-49742015000100019

Gavegnano, C., Haile, W. B., Hurwitz, S., Tao, S., Jiang, Y., Schinazi, R. F., \& Tyor, W. R. (2019). Baricitinib reverses HIV-associated neurocognitive disorders in a SCID mouse model and reservoir seeding in vitro. Journal of Neuroinflammation, 16(1), 182. https://doi.org/10.1186/s12974-019-1565-6

Geffin, R., \& McCarthy, M. (2018). Aging and Apolipoprotein E in HIV Infection. Journal of Neurovirology, 24(5), 529-548. https://doi.org/10.1007/s13365018-0660-2

Gelman, B. B., Lisinicchia, J. G., Morgello, S., Masliah, E., Commins, D., Achim, C. L., Fox, H. S., Kolson, D. L., Grant, I., Singer, E., Yiannoutsos, C. T., Sherman, S., Gensler, G., Moore, D. J., Chen, T., \& Soukup, V. M. (2013). Neurovirological correlation with HIV-associated neurocognitive disorders and encephalitis in a HAART-era cohort. Journal of Acquired Immune Deficiency Syndromes (1999), 62(5), 487-495. https://doi.org/10.1097/QAI.0b013e31827f1bdb

Gendelman, H. E., \& Gelbard, H. A. (2014). Adjunctive and long-acting nanoformulated antiretroviral therapies for HIV-associated neurocognitive disorders. Current Opinion in HIV and AIDS, 9(6), 585-590. https://doi.org/10.1097/COH.0000000000000111

Han, H., Yang, Z., Chang, S. L., \& Li, M. D. (2018). Modulatory Effects of Nicotine on neuroHIV/neuroAIDS. Journal of Neuroimmune Pharmacology : The Official Journal of the Society on NeuroImmune Pharmacology, 13(4), 467-478. https://doi.org/10.1007/s11481-018-9806-5

Illenberger, J. M., Harrod, S. B., Mactutus, C. F., McLaurin, K. A., Kallianpur, A., \& Booze, R. M. (2020). HIV Infection and Neurocognitive Disorders in the Context of Chronic Drug Abuse: Evidence for Divergent Findings Dependent upon Prior Drug History. Journal of Neuroimmune Pharmacology : The Official Journal of the Society on NeuroImmune Pharmacology, 15(4), 715-728. https://doi.org/10.1007/s11481-020-09928-5

Liu, F., Dai, S., Gordon, J., \& Qin, X. (2014). Complement and HIV-I infection/HIV-associated neurocognitive disorders. Journal of Neurovirology, 20(2), 184198. https://doi.org/10.1007/s13365-014-0243-9

Mackiewicz, M. M., Overk, C., Achim, C. L., \& Masliah, E. (2019). Pathogenesis of age-related HIV neurodegeneration. Journal of Neurovirology, 25(5), 622633. https://doi.org/10.1007/s13365-019-00728-z

Mohamed, A. A., Oduor, C., \& Kinyanjui, D. (2020). HIV-associated neurocognitive disorders at Moi teaching and referral hospital, Eldoret, Kenya. BMC Neurology, 20(1), 280. https://doi.org/10.1186/s12883-020-01857-3

Namagga, J. K., Rukundo, G. Z., \& Voss, J. G. (2019). Prevalence and Risk Factors of HIV-Associated Neurocognitive Disorders in Rural Southwestern Uganda. The Journal of the Association of Nurses in AIDS Care : JANAC, 30(5), 531-538. https://doi.org/10.1097/JNC.0000000000000036

Neves, M. P. das, Silva, R. R. da, Silva, L. A. da, Silva, M. V. G. da, Silva, M. M. dos S. da, Francisco, M. T. R., \& Marta, C. B. (2019). Adição à drogas, o consumo de substâncias psicoativas por jovens, utilizando o instrumento assist. Saúde Coletiva (Barueri), 51, 1913-1919. https://doi.org/10.36489/saudecoletiva.2019v9i51p1913-1919

Page, M. J., Moher, D., Bossuyt, P. M., Boutron, I., Hoffmann, T. C., Mulrow, C. D., Shamseer, L., Tetzlaff, J. M., Akl, E. A., Brennan, S. E., Chou, R., Glanville, J., Grimshaw, J. M., Hróbjartsson, A., Lalu, M. M., Li, T., Loder, E. W., Mayo-Wilson, E., McDonald, S., ... McKenzie, J. E. (2021). PRISMA 2020 explanation and elaboration: updated guidance and exemplars for reporting systematic reviews. BMJ, n160. https://doi.org/10.1136/bmj.n160

Pulliam, L., Sun, B., Mustapic, M., Chawla, S., \& Kapogiannis, D. (2019). Plasma neuronal exosomes serve as biomarkers of cognitive impairment in HIV infection and Alzheimer's disease. Journal of Neurovirology, 25(5), 702-709. https://doi.org/10.1007/s13365-018-0695-4

Rahimian, P., \& He, J. J. (2017). HIV/neuroAIDS biomarkers. Progress in Neurobiology, 157, 117-132. https://doi.org/10.1016/j.pneurobio.2016.04.003

Rosenthal, J., \& Tyor, W. (2019). Aging, comorbidities, and the importance of finding biomarkers for HIV-associated neurocognitive disorders. Journal of Neurovirology, 25(5), 673-685. https://doi.org/10.1007/s13365-019-00735-0

Ru, W., \& Tang, S.-J. (2017). HIV-associated synaptic degeneration. Molecular Brain, 10(1), 40. https://doi.org/10.1186/s13041-017-0321-z

Rubin, L. H., Sundermann, E. E., \& Moore, D. J. (2019). The current understanding of overlap between characteristics of HIV-associated neurocognitive disorders and Alzheimer's disease. Journal of Neurovirology, 25(5), 661-672. https://doi.org/10.1007/s13365-018-0702-9

Sacktor, N. (2018). Changing clinical phenotypes of HIV-associated neurocognitive disorders. Journal of Neurovirology, 24(2), 141-145. https://doi.org/10.1007/s13365-017-0556-6

Sacktor, N., Skolasky, R. L., Seaberg, E., Munro, C., Becker, J. T., Martin, E., Ragin, A., Levine, A., \& Miller, E. (2016). Prevalence of HIV-associated neurocognitive disorders in the Multicenter AIDS Cohort Study. Neurology, 86(4), 334-340. https://doi.org/10.1212/WNL.0000000000002277

Silva, R. R. da, Lipari, C. da C., Araujo, M. S., Silva, L. A. da, Silva, M. V. G. da, Franco, A. S., Marta, C. B., Larrubia, E. de O., Francisco, M. T. R., \& Oliveira, E. S. de. (2021). Contribuições da Monitoria em Fundamentos de Enfermagem II na Formação Acadêmica de Estudantes de Enfermagem: Relato de Experiência. Global Academic Nursing Journal, 2(1). https://doi.org/10.5935/2675-5602.20200079 
Research, Society and Development, v. 11, n. 2, e47311226039, 2022

(CC BY 4.0) | ISSN 2525-3409 | DOI: http://dx.doi.org/10.33448/rsd-v11i2.26039

Silva, R. R. da, Neves, M. P. das, Silva, L. A. da, Silva, M. V. G. da, Hipolito, R. L., \& Marta, C. B. (2020). Consumo de Drogas Psicoativas em Contexto de Sexual entre Homens Gays como Fator de Risco para Transmissão de HIV/Aids. Global Academic Nursing Journal, 1(3). https://doi.org/10.5935/26755602.20200057

Silva, R. R. da, \& Silva, L. A. da. (2021). Psychosocial load and burnout syndrome in healthcare professionals in the fight against COVID-19 pandemic / Carga psicossocial e síndrome de burnout em profissionais de saúde no combate a pandemia de COVID-19. Revista de Pesquisa Cuidado é Fundamental Online, 13, 1640-1646. https://doi.org/10.9789/2175-5361.rpcfo.v13.11097

Silva, R. R. da, Silva, L. A. da, Silva, M. V. G. da, Neves, M. P. das, Silva, M. M. dos S. da, Francisco, M. T. R., \& Marta, C. B. (2019). Os impactos do Chemsex na saúde pública mundial: um estudo sobre uma perigosa prática sexual entre homens. Saúde Coletiva (Barueri), 51, 1920-1925. https://doi.org/10.36489/saudecoletiva.2019v9i51p1920-1925

Silva, R. R. da, Silva, L. A. da, Souza, M. V. L. de, Silva, M. V. G. da, Neves, M. P. das, Vargas, D. de, Hipolito, R. L., Souza, D. A. C., Dutra, V. de C. de A., Oliveira, E. S. de, Lipari, C. da C., Garcia, W., Cortes, T., \& Mattos, C. M. (2021). Estresse de minoria de gênero e seus efeitos na saúde mental como fator de risco para depressão em pessoas transgênero: Revisão da literatura. Research, Society and Development, 10(3), e51610313693. https://doi.org/10.33448/rsdv10i3.13693

Silva, R. R., Pontes, L. G. de, Oliveira, G. A. de, Assmann, T. C., Campos, E. da C., Silva, A. A. da, \& Souza, M. V. L. de. (2021). Avaliação dos fatores de risco e diagnóstico para neuropatia autonômica cardíaca em pessoas diabéticas. Global Academic Nursing Journal, 2(nspe3). https://doi.org/10.5935/26755602.20200164

Souza, M. V. L. de, Silva, R. R. da, Oliveira, M. C. P. de, Silva, L. A. da, Silva, M. V. G. da, Vargas, D. de, Hipólito, R. L., Souza, M. das G. G. de, Silveira, M. L. F. G. da, Mesquita, L. M. F. de, Araújo, M. S., Ignácio, L. P., Fontes, T. V., Alencar, Í. F. de, Souza, D. A. C., Oliveira, J. V. E., Neves, M. P. das, Pereira, A. V., Soares Filho, M. O., \& Dutra, V. de C. de A. (2021). Acesso a PrEP por homens cisgênero e transexuais: Um estudo de abordagem qualitativa. Research, Society and Development, 10(1), e44310111843. https://doi.org/10.33448/rsd-v10i1.11843

Souza, M. T. de, Silva, M. D. da, \& Carvalho, R. de. (2010). Integrative review: what is it? How to do it? Einstein (São Paulo), 8(1), 102-106. https://doi.org/10.1590/s1679-45082010rw1134

Thaney, V. E., \& Kaul, M. (2019). Type I Interferons in NeuroHIV. Viral Immunology, 32(1), 7-14. https://doi.org/10.1089/vim.2018.0085

Troncoso, F. T., \& Conterno, L. de O. (2015). Prevalence of neurocognitive disorders and depression in a Brazilian HIV population. Revista Da Sociedade Brasileira de Medicina Tropical, 48(4), 390-398. https://doi.org/10.1590/0037-8682-0034-2015

Tyor, W. R., \& Bimonte-Nelson, H. (2018). A mouse model of HIV-associated neurocognitive disorders: a brain-behavior approach to discover disease mechanisms and novel treatments. Journal of Neurovirology, 24(2), 180-184. https://doi.org/10.1007/s13365-017-0572-6

Tyor, W., Fritz-French, C., \& Nath, A. (2013). Effect of HIV clade differences on the onset and severity of HIV-associated neurocognitive disorders. Journal of Neurovirology, 19(6), 515-522. https://doi.org/10.1007/s13365-013-0206-6

Vázquez-Santiago, F. J., Noel, R. J. J., Porter, J. T., \& Rivera-Amill, V. (2014). Glutamate metabolism and HIV-associated neurocognitive disorders. Journal of Neurovirology, 20(4), 315-331. https://doi.org/10.1007/s13365-014-0258-2 